\title{
Transtorno de estresse pós-traumático decorrente de acidente de trabalho: implicações psicológicas, socioeconômicas e jurídicas
}

\author{
Luiziana Souto Schaefer \\ Beatriz de Oliveira Meneguelo Lobo \\ Christian Haag Kristensen \\ Pontificia Universidade Católica do Rio Grande do Sul
}

\begin{abstract}
Resumo
Entre as possíveis consequências dos acidentes de trabalho ressalta-se que, embora os prejuízos físicos sejam mais facilmente percebidos, sintomas e transtornos psiquiátricos têm sido cada vez mais observados. Nesse contexto, o Transtorno de Estresse Pós-Traumático (TEPT) é um dos transtornos mais comuns, causando prejuízos para os trabalhadores e tendo grande impacto na sua qualidade de vida. O objetivo deste artigo foi apresentar uma revisão teórica sobre o TEPT decorrente de acidente de trabalho, bem como revisar as implicações psicológicas, sócio-econômicas e jurídicas desencadeadas pelo transtorno. A partir da revisão da literatura apresentada, fica evidente a repercussão do TEPT para a vida do indivíduo, com prejuízos significativos nas relações familiares, sociais e, sobretudo, no ambiente de trabalho. Finalmente, a discussão sobre o TEPT no contexto do trabalho suscita para a consideração em torno de diferentes eixos: prevenção, avaliação, tratamento e ressarcimento acerca dos prejuízos causados.
\end{abstract}

Palavras-chave: transtorno de estresse pós-traumático; acidentes de trabalho; saúde do trabalhador.

\begin{abstract}
Posttraumatic stress disorder resulting from occupational accident: psychological, socioeconomic, and legal implications. Among the possible consequences of occupational accidents, it is emphasized that although the physical damage are more easily perceived, psychiatric symptoms and disorders have been increasingly observed. In this context, Post-Traumatic Stress Disorder (PTSD) is one of the most common, causing injuries to workers and having great impact on their quality of life. The aim of this paper was to present a theoretical review of PTSD resulting from occupational accidents as well as review the psychological, socioeconomic and legal consequences triggered by the disorder. From the review of the literature presented, it is evident the impact of PTSD for the individual's life, with significant losses in their relationships and in the workplace. Finally, the discussion about PTSD in the work environment raises for consideration about prevention, assessment, treatment and compensation.
\end{abstract}

Keywords: post-traumatic stress disorders; occupational accidents; occupational health.

$\mathrm{D}$ urante os séculos XVIII e XIX, ocorreram transformações socioeconômicas cruciais na sociedade devido ao advento da Revolução Industrial, trazendo repercussões significativas ao ambiente de trabalho e à saúde das pessoas, sobretudo dos trabalhadores. Nesse período, o aumento da produção foi priorizado em detrimento das condições de trabalho e da saúde dos trabalhadores submetidos, frequentemente, a um processo de produção acelerado e desumano (Mendes \& Dias, 1991; Murofuse \& Marziale, 2001; Seligmann-Silva, 1997). Infelizmente, essa postura ainda é observada nos ambientes organizacionais, visto que a maioria dos empregadores demonstra interesse na promoção da saúde apenas quando esta interfere na produtividade dos trabalhadores e, consequentemente, impacta negativamente os rendimentos da organização.

Além disso, é frequente a ocorrência de acidentes de trabalho no ambiente organizacional. Tais experiências são consideradas um fator importante para o desenvolvimento do estresse e incluem qualquer tipo de acidente que ocorra pelo exercício do trabalho e que provoque lesão corporal ou perturbação funcional, de caráter temporário ou permanente. Essa lesão ou perturbação pode provocar morte, perda ou redução da capacidade para o trabalho (Brasil, 1991).

Entre as possíveis consequências dos acidentes de trabalho, ressalta-se que, embora os prejuízos físicos sejam mais facilmente percebidos, sintomas e transtornos psiquiátricos têm sido cada vez mais observados (Ministério da Saúde do Brasil, 2001). Nesse contexto, o Transtorno de Estresse Pós-Traumático (TEPT) vem sendo apontado como uma das psicopatologias que podem ser desencadeadas após a vivência de um acidente no contexto do trabalho, causando prejuízos 
e impactos na qualidade de vida dos trabalhadores (Bucasio et al., 2005; Buodo et al., 2011; McFarlane \& Bryant, 2007; Novara et al., 2009; World Health Organization, 1998). Embora haja categorias profissionais mais suscetíveis a desenvolver o TEPT, como bancários, policiais, enfermeiros, bombeiros, entre outros, o transtorno não se restringe a esses profissionais, podendo acometer qualquer trabalhador, tendo em vista que a ocorrência de situações traumáticas não é um fato incomum no ambiente organizacional (MacDonald, Colotla, Flamer, \& Karlinsky, 2003; McFarlane \& Bryant, 2007). Este artigo teve como principal objetivo apresentar uma revisão não sistemática da literatura sobre o TEPT decorrente de acidente de trabalho, bem como as implicações psicológicas, socioeconômicas e jurídicas desencadeadas pelo transtorno.

\section{Acidente de trabalho}

São inúmeras as situações vivenciadas durante a jornada de trabalho que podem ser consideradas traumáticas e, consequentemente, impactar a saúde física e mental das vítimas. Entre essas situações, denominadas de acidentes de trabalho, incluem-se: explosões, queimaduras, choques elétricos, assaltos, agressões, acidentes industriais, amputação de membros, entre outras (Foa, Keane, \& Friedman, 2000; McFarlane \& Bryant, 2007; MacDonald et al., 2003).

Conforme a Lei 8.213 de 24 de julho de 1991, artigo 19 (Brasil, 1991), "acidente do trabalho é o que ocorre pelo exercício do trabalho a serviço da empresa ou pelo exercício do trabalho do segurado especial, provocando lesão corporal ou perturbação funcional, que cause a morte ou a perda ou redução, permanente ou temporária, da capacidade para o trabalho", sendo responsabilidade do empregador a "adoção e uso das medidas coletivas e individuais de proteção e segurança do trabalhador". Ainda, equiparam-se a acidente de trabalho: o acidente relacionado ao trabalho que haja contribuído diretamente para a ocorrência da lesão, embora não tenha sido a causa única; o acidente sofrido a serviço da empresa ou no trajeto entre a residência e o local de trabalho do segurado e vice-versa; certos acidentes sofridos pelo segurado no local e no horário de trabalho, como atos de agressão, sabotagem, terrorismo, ofensa física intencional, desabamento, incêndio, entre outros (Brasil, 1991). Portanto, para o reconhecimento da natureza acidentária, é suficiente que o ambiente de trabalho tenha contribuído para o desenvolvimento ou agravamento do quadro clínico, não sendo necessário configurar-se como fator único ou predominante.

Segundo o Anuário Estatístico de Acidentes do Trabalho de 2008 (Ministério do Trabalho e Emprego \& Ministério da Previdência Social, 2009), foram registrados, naquele ano, 747.663 acidentes de trabalho no Brasil, entre os trabalhadores segurados da Previdência Social, com uma taxa de incidência de 22,94 (por 1.000 vínculos). A maior incidência constatada foi no estado de Santa Catarina (28,04 em 2007 e 31,78 em 2008), seguido por Alagoas (29,82 em 2007 e 31,72 em 2008) e Rio Grande do Sul (26,36 em 2007 e 29,48 em 2008). É importante ressaltar que no ano de 2008, 202.395 trabalhadores não tiveram a Comunicação de Acidente de Trabalho (CAT) registrada (Ministério do Trabalho e Emprego \& Ministério da Previdência Social, 2008; 2009). Os dados também permitem observar que, no Brasil, em 2007, houve uma média de 31 trabalhadores/dia que não retornaram mais ao trabalho por invalidez ou morte. Além disso, a cada três horas ocorreu cerca de uma morte no ambiente de trabalho e, ainda, cerca de 75 acidentes e doenças do trabalho a cada uma hora na jornada diária (Ministério da Previdência Social, 2010). Com relação aos benefícios acidentários, em 2008, o INSS concedeu 377.001 novos benefícios, entre auxílios-doença e aposentadorias por invalidez decorrentes de acidentes de trabalho. Destes, 13.078 foram por Transtornos Mentais e Comportamentais, representando a terceira maior causa de concessão de auxílios-doença acidentários (Ministério da Previdência Social, Empresa de Tecnologia e Informações da Previdência Social, 2009).

Com a implementação do Nexo Técnico Epidemiológico Previdenciário (NTEP), em abril de 2007, os registros de acidentes de trabalho aumentaram significativamente, contribuindo para reduzir a subnotificação (Ministério da Previdência Social, Empresa de Tecnologia e Informações da Previdência Social, 2009). Com o NTEP, a doença cuja incidência for elevada em determinada atividade econômica será caracterizada como relacionada ao trabalho, independentemente da emissão da CAT, ou seja, o nexo causal é presumido à categoria profissional, cabendo à empresa o ônus da prova (Brasil, 2006).

Especificamente sobre os transtornos elencados pela Classificação dos Transtornos Mentais e de Comportamento da CID-10 (Organização Mundial da Saúde, 1993) no grupo de Reação ao Estresse Grave e Transtornos de Ajustamento (F43), entre os quais se encontra o TEPT (F43.1), foram registrados 3.037 casos de acidente de trabalho no ano de 2006, 5.278 casos em 2007, 7.026 casos em 2008 (Ministério do Trabalho e Emprego \& Ministério da Previdência Social, 2009). Tais achados apontam para o número crescente de casos registrados pertencentes a esta categoria, evidenciando a grande quantidade de acidentes de trabalho classificados no grupo de estresse. Ao mesmo tempo, pode-se pensar que tais dados estejam aquém da realidade, tendo em vista que muitos transtornos mentais acabam não sendo diagnosticados, principalmente pela ausência de lesão física aparente. Além disso, essas informações referemse apenas aos trabalhadores contratados pelo regime da CLT, não incluindo, por exemplo, servidores públicos, profissionais liberais, autônomos, militares, trabalhadores informais, pequenos comerciantes, entre outros.

Entre as consequências para os trabalhadores que vivenciam um acidente de trabalho, a literatura aponta: desajuste emocional, irritabilidade, hostilidade, episódios depressivos, transtornos cognitivos, transtornos do sono, alcoolismo crônico, entre outros e, sobretudo, o TEPT (Cohidon et al., 2009; Jacques, 2007; Ministério da Saúde do Brasil, 2001; Steffgen, 2008). Assim, é possível perceber que experienciar um evento traumático durante a jornada de trabalho pode ser categorizado como acidente de trabalho, repercutindo negativamente tanto na vida da vítima quanto no próprio ambiente de trabalho.

\section{Transtorno de Estresse Pós-Traumático}

O TEPT é o transtorno psicológico mais comum após a experiência de um evento estressor traumático (Keane, Marshall, \& Taft, 2006). A quarta edição revisada do Manual Diagnóstico 
e Estatístico de Transtornos Mentais (DSM-IV-TR; Associação Americana de Psiquiatria [APA], 2002) refere ampla gama de eventos estressores traumáticos que podem desencadear o TEPT, como por exemplo: sequestro, assalto à mão armada, desastres naturais ou causados pelo homem, acidentes automobilísticos graves, tortura, ataque sexual ou físico, entre outros. Tais situações são experienciadas com frequência, já que estudos epidemiológicos estimam que mais de metade da população geral enfrentará ao menos um evento traumático ao longo de suas vidas (Kessler, Sonnega, Bromet, Hughes, \& Nelson, 1995). Por exemplo, no estudo de revisão realizado por Sher (2004), estimou-se que entre 40 a $90 \%$ das pessoas serão expostas a eventos estressores traumáticos durante a vida. Ainda, expor-se a mais de um trauma ao longo da vida aumenta as chances de desenvolver TEPT (Breslau, Chilcoat, Kessler, \& Davis, 1999; Green et al., 2010).

No National Comorbidity Survey (Kessler, Berglund et al., 2005, Kessler, Chiu, Demler, Merikangas, \& Walters, 2005), o TEPT foi considerado o quinto transtorno mental mais prevalente nos últimos 12 meses, com uma prevalência do transtorno ao longo da vida estimada em 6,8\%. Dessa maneira, é possível constatar que, apesar da maioria das pessoas expostas a eventos potencialmente traumáticos não desenvolverem o TEPT, a prevalência do transtorno ainda é bastante elevada, considerando que estas estimativas referem-se à população geral.

O TEPT é definido, no DSM-IV-TR (APA, 2002), como um conjunto de sintomas desenvolvidos pelo indivíduo após a exposição a um estressor traumático, no qual os seguintes critérios diagnósticos devem estar presentes: a pessoa experienciou, testemunhou ou foi confrontada com um ou mais eventos que envolveram morte, sério ferimento ou outra ameaça à integridade física, própria ou de outros (critério A1) e cuja reação envolveu intenso medo, impotência ou horror (critério A2). A partir disso, o indivíduo apresenta uma resposta sintomática envolvendo revivência contínua do evento traumático (critério B), evitação e entorpecimento (critério C) e excitabilidade aumentada (critério D), sendo que a duração desses sintomas é superior a um mês (critério E) e causa sofrimento clinicamente significativo ou prejuízo em áreas importantes da vida do indivíduo (critério F).

Entre os sintomas de revivência, incluem-se: pensamentos intrusivos que invadem a consciência sem o consentimento do indivíduo, sonhos aflitivos e recorrentes relacionados à experiência traumática vivenciada, sensação de que o evento está ocorrendo novamente, sofrimento psicológico intenso e/ ou reatividade fisiológica quando exposto a estímulos que lembrem algum aspecto do trauma. No critério de esquiva e entorpecimento, as respostas mais comuns dizem respeito a tentativas de evitar pensamentos, atitudes, sentimentos, locais e/ou pessoas relacionadas ao trauma, incapacidade de lembrar algum aspecto importante do evento traumático, perda de interesse por atividades anteriormente prazerosas, sensação de distanciamento das outras pessoas e/ou de um futuro abreviado. Por último, entre a manifestação sintomática incluída no critério de excitabilidade aumentada, é comum a presença de: irritabilidade, dificuldades de concentração, hipervigilância (estar em alerta constante), reação de sobressalto exagerada e dificuldades em conciliar ou manter o sono (APA, 2002).
O TEPT é um transtorno que traz inúmeras mudanças para a vida de suas vítimas, que acabam constantemente revivendo a situação traumática e apresentando reações extremas de ansiedade como se o evento estivesse continuamente ocorrendo. Ademais, grande parte da literatura sobre o transtorno aborda vítimas de eventos traumáticos específicos e, mais recentemente, percebe-se um interesse crescente nos estudos sobre TEPT nos contextos de trabalho (MacDonald et al., 2003).

TEPT decorrente de acidente de trabalho. O trabalho deveria ser um fator de promoção da saúde, visto que por intermédio dele o indivíduo se desenvolve nas dimensões psicológica, social e econômica (Seligmann-Silva, 1997). No entanto, para aqueles trabalhadores que experienciam situações traumáticas durante a jornada de trabalho, torna-se nocivo para o empregado, especialmente quando realizado sob condições materiais e psicológicas inadequadas. Diante disso, a atividade profissional perde o significado para o trabalhador, de modo que não favorece o seu desenvolvimento pessoal, levando a um esgotamento (Palácios, Duarte, \& Câmara, 2002; SeligmannSilva, 1997).

O TEPT é um transtorno comumente relacionado ao trabalho (Berninger et al. 2010; Hensel, Bender, Bacchiochi, Pelletier, \& Dewa, 2010; McFarlane \& Bryant, 2007; Shercliffe \& Colotla, 2009), juntamente com outros transtornos de ansiedade, estresse ocupacional, depressão e síndrome de burnout (World Health Organization, 1998). No Brasil, é adotada a classificação de Schilling para sistematizar as doenças relacionadas ao trabalho, as quais podem ser distribuídas em três grupos principais: grupo I (o trabalho é considerado como causa necessária para o aparecimento da doença), o grupo II (o trabalho pode contribuir para o desenvolvimento da doença, porém não é fator necessário) e o grupo III (o trabalho configura-se como um provocador de um distúrbio latente ou agravador de doença já estabelecida ou preexistente). Assim, as doenças classificados nos grupos II ou III são consideradas de etiologia múltipla e o trabalho seria entendido como um fator de risco, sendo o nexo causal, portanto, de natureza essencialmente epidemiológica (Ministério da Saúde do Brasil, 2001; Teixeira, 2007). Segundo esta classificação, a maioria dos transtornos mentais e de comportamento estaria classificada no grupo III. Entretanto, entre algumas exceções encontra-se o TEPT, classificado no grupo I, e, nesse caso, excluídas outras causas não ocupacionais, o trabalho ou as condições em que ele é realizado constituem causa direta, necessária e imediata para o surgimento da doença (Ministério da Saúde do Brasil, 2001).

Em um estudo de revisão, foi constatado que de 10 a $18 \%$ dos casos de violência no trabalho irão desenvolver sintomas que satisfazem os critérios para o TEPT (Steffgen, 2008). Um estudo semelhante observou que $44 \%$ dos trabalhadores que passaram por um acidente de trabalho preenchiam critérios para TEPT (Hensel et al., 2010). Ainda, 28,9\% dos indivíduos que passaram por este tipo de trauma apresentam sintomas moderados a severos e 10,5\% apresentavam sintomatologia severa de TEPT (Buodo et al., 2011).

A literatura descreve vítimas de TEPT decorrente dos mais variados tipos de acidentes de trabalho, a saber: incêndios, queimaduras (Van Loey, Son, Heijden, \& Ellis, 2008; Wolf, 
2009), amputação de membros do corpo (Buodo et al., 2011), agressão física e/ou psicológica, assaltos (Bucasio et al., 2005; De Mol, 1998; Dorigo \& Lima, 2007; Richards, 2000), sequestros (Hernández, Gangsel, \& Engstrom, 2007), acidentes industriais (Shercliffe \& Colotla, 2009), entre outros. Além disso, entre os grupos profissionais comumente atingidos pelo TEPT, encontram-se: policiais, bombeiros, profissionais da área da saúde e bancários (Berger et al., 2007; Berninger et al. 2010; Einav et al., 2008; MacDonald et al., 2003; Maia et al., 2007; Wells et al., 2008). Para ilustrar, uma pesquisa realizada entre 2001 e 2005 com 1074 bombeiros demonstrou que 9,8 a 10,6\% satisfizeram critérios para um elevado risco de desenvolver TEPT durante os quatro anos do estudo (Berninger et al. 2010). No Brasil, verificou-se que, entre 234 trabalhadores em ambulâncias, $5,6 \%$ preenchiam critérios diagnósticos para o TEPT e $15 \%$ apresentavam sintomas pós-traumáticos (Berger et al., 2007).

O trabalhador pode desenvolver o TEPT como um diagnóstico adicional, em decorrência de um acidente de trabalho específico, como nas situações em que tem parte de seu corpo amputada pela utilização de uma determinada máquina, ou ele mesmo ser a única consequência do acidente de trabalho, como nas ocasiões em que vivencia um assalto à mão armada sem qualquer tipo de lesão física (MacDonald et al., 2003). Em virtude de tais possibilidades, percebe-se que, nos casos em que há um dano físico, a constatação do acidente de trabalho fica mais evidente. Por outro lado, muitas vezes os diagnósticos psiquiátricos não são realizados e, consequentemente, acabam sendo subestimados.

Especificamente em relação ao TEPT nos contextos de trabalho, foi realizado um estudo com 44 trabalhadores portadores do transtorno cujos pedidos foram aceitos para a concessão de benefícios de compensação, na ausência de lesões físicas significativas (MacDonald et al., 2003). Entre esses, $82 \%$ vivenciaram diretamente o evento traumático e os demais foram testemunhas. Tal fato demonstra que os transtornos psiquiátricos vêm tomando espaço nas discussões científicas e, em decorrência disso, explicitam a necessidade de que a legislação seja constantemente repensada.

No Brasil, o TEPT ainda é um transtorno relativamente pouco diagnosticado e pouco estudado, dificultando, portanto, o reconhecimento do diagnóstico de TEPT relacionado a acidente de trabalho (Bucasio et al., 2005). Entre as prováveis justificativas para isso, ressalta-se a falta de conhecimento desse transtorno - não somente entre a população geral, como também entre os profissionais da saúde - bem como a alta resistência à busca de tratamento presente entre os pacientes com esse diagnóstico (Bucasio et al., 2005), possivelmente devido aos próprios sintomas de evitação característicos do transtorno. Em decorrência disso, muitos indivíduos que desenvolvem o TEPT continuam a sofrer indefinidamente, demorando a buscar auxílio, cronificando o transtorno e tornando o tratamento ainda mais difícil (Wells et al., 2008).

\section{Implicações psicológicas, socioeconômicas e jurídi- cas}

São visíveis as implicações do TEPT para a saúde e o bem-estar social dos indivíduos. Do ponto de vista psicológico, alterações de humor, irritabilidade e agressividade podem ser sintomas desencadeados pelo transtorno (Monson \& Friedman, 2006; Taylor, 2006), assim como alterações no sono (Lydiard \& Hamner, 2009) e em funções cognitivas (Brewin, 2007; Buodo et al., 2011; Rubin, Berntsen, \& Johansen, 2008), uso de substâncias psicoativas (Dantas \& Andrade, 2008), entre outras.

No estudo de Kessler e colaboradores (1995), constatou-se que pelo menos $80 \%$ dos indivíduos com TEPT preenchiam, adicionalmente, critérios diagnósticos para outras psicopatologias. A literatura aponta elevada comorbidade entre TEPT e outros transtornos de ansiedade, como o Transtorno de Pânico, Agorafobia, Transtorno Obsessivo-Compulsivo, Fobia Social e Fobias Específicas, transtornos de humor, como o Transtorno Depressivo Maior, além de risco de suicídio, uso de substâncias químicas, como o álcool e outras drogas, e jogo patológico (Cacciola, Koppenhaver, Alterman, \& McKay, 2009; Kessler, 2000; Kessler et al., 2008; Kristensen, Parente, \& Kaszniak, 2005; Nock et al., 2009; Ouimette, Read, Wade, \& Tirone, 2010). Em estudo com trabalhadores que desenvolveram TEPT nos contextos do trabalho, foi constatado que $45 \%$ dos participantes possuíam pelo menos outra psicopatologia, sendo que a depressão foi a mais comórbida (MacDonald et al., 2003). A alta prevalência de comorbidades suscita, num primeiro momento, para dificuldades em termos de constatação diagnóstica e, posteriormente, para a elaboração do plano de tratamento, uma vez que transtornos mentais mais conhecidos podem acabar sendo confundidos ou avaliados primeiramente, mascarando o diagnóstico de TEPT (Knapp \& Caminha, 2003; Margis, 2003).

Além dos prejuízos físicos e emocionais ocasionados pelo TEPT, a literatura descreve, ainda, consequências sociais e econômicas significativas (Beck, Grant, Clapp, \& Palyo, 2009; Keane et al., 2006; Olatunji, Cisler, \& Tolin, 2007; Taylor, 2006). No Brasil, ainda não há registros suficientes que permitam estabelecer de maneira precisa os gastos anuais despendidos com o TEPT. Nos Estados Unidos, Kessler (2000) estimou que pessoas com TEPT perdem cerca de 3,6 dias de trabalho por mês, o que significa um prejuízo na produtividade anual acima de US\$ 3 bilhões nos Estados Unidos. Já os gastos destinados à saúde anualmente por pessoa com alta sintomatologia de TEPT representam, em média, US\$ 1283.00 para o país (Walker et al., 2003). Indivíduos com TEPT têm risco aumentado para desenvolver problemas médicos e, assim, utilizam mais o sistema de saúde (Olatunji et al., 2007; Schnurr, Green, \& Kaltman, 2007).

Os sintomas de TEPT também podem interferir significativamente nos relacionamentos e nas atividades ocupacionais (Matthews, Harris, \& Cumming, 2009; Taylor, 2006). A evitação, o retraimento social, a irritabilidade e a agressividade resultam em distanciamento da família e amigos; o entorpecimento prejudica a comunicação; a excitabilidade aumentada resulta em dificuldades de concentração, fadiga, entre outros (Kessler, 2000; Taylor, 2006).

O TEPT decorrente de acidente de trabalho pode acarretar numa diminuição no nível de satisfação do trabalho, além de altos índices de absenteísmo, rotatividade de funcionários, licenças para tratamento de saúde e, até mesmo, aposentadoria adiantada (Bucasio et al., 2005; Hensel et al., 2010; Matthews et 
al., 2009; Miller, 2001). Além do afastamento para o tratamento, o TEPT implica num projeto de reabilitação profissional, já que possíveis decorrências, como por exemplo, a persistência de quadros fóbicos pode acarretar num empecilho para o retorno ao posto de trabalho anteriormente ocupado (Ministério da Saúde do Brasil, 2001). A partir disso, percebe-se grande interesse dos empregadores às questões referentes à saúde, particularmente quando estas interferem na produtividade e trazem inúmeras implicações econômicas (Chamon, 2006).

No momento em que ocorre um acidente de trabalho, cabe ao empregador a emissão da CAT até o primeiro dia útil seguinte ao da ocorrência do acidente, sob pena de multa, conforme prevê o artigo 22 da Lei 8.213/91 (Brasil, 1991). Com relação à doença decorrente de acidente de trabalho, compete à jurisprudência o julgamento diante dos fatos probatórios apresentados no processo. Do ponto de vista jurídico, quando estabelecido o nexo causal entre a doença e o trabalho, o empregado tem assegurado direitos previdenciários e trabalhistas, podendo ajuizar ações judiciais autônomas. Em decorrência disso, poderão ser concedidos ao trabalhador, mediante perícia médica junto ao INSS que avalia o desenvolvimento do quadro clínico, benefícios como: auxílio-doença, auxílio-acidente, aposentadoria por invalidez e pensão por morte (Teixeira, 2007). Além disso, o segurado também tem direito à estabilidade acidentária provisória, pelo prazo mínimo de doze meses, após a alta previdenciária (Brasil, 1991). Conforme previsto no inciso XXVIII do art. $7^{\circ}$ da Constituição Federal (CF, Brasil, 1988) e nos artigos 186 e 927 do Código Civil Brasileiro (Brasil, 2002), o trabalhador também pode ajuizar, na Justiça trabalhista, ação indenizatória por danos morais e materiais decorrentes do infortúnio laboral (Teixeira, 2007).

A responsabilidade penal ocorre quando o agente viola norma de direito público. Já quando descumpre norma que tutela interesse privado, causando um dano indenizável, a responsabilidade é civil (Remédio, Freitas, \& Junior, 2000). No ordenamento jurídico brasileiro, especificamente no conceito analítico do crime, a consideração do nexo causal exerce papel central na determinação de uma conduta humana como típica, ilícita (antijurídica) e culpável, ou seja, criminosa (Bruno, 1984 citado por Duarte, 2002). No artigo 186 do Código Civil (Brasil, 2002), é dito que "aquele que, por ação ou omissão voluntária, negligência ou imprudência, violar direito e causar dano a outrem, ainda que exclusivamente moral, comete ato ilícito", sendo que os pressupostos deste ato incluem a ação ou omissão do agente, o dolo ou a culpa, o dano e, por fim, o nexo causal entre o dano e a ação (Remédio et al., 2000). De forma semelhante, a conditio sine qua non (causalidade) tem fundamental importância no estabelecimento de determinado fato como acidente de trabalho e, mais especificamente, na consignação de tal ocorrência como condição estritamente necessária para o desencadeamento de psicopatologias relacionadas a acidentes de trabalho (Dorigo \& Lima, 2007; Jacques, 2007; Lima, 2005; Vieira, 2009).

Nos acidentes de trabalho que ocasionam traumas físicos, como amputação de membros pela utilização de uma máquina, as consequências e os danos trazidos para o indivíduo são evidentes. Entretanto, em circunstâncias nas quais não há uma lesão física aparente, como em situações de assalto à mão armada sem agressão física, os prejuízos são menos explícitos, embora possam também estar presentes, visto que o indivíduo pode sofrer um dano psíquico ocasionado pela vivência do trauma.

Diante disso, convém ressaltar as diferenças entre os conceitos de dano moral e dano psíquico. O primeiro diz respeito à presença de uma dor ou sofrimento decorrente da violação de algum dos direitos inerentes à personalidade, sendo constatado por agentes jurídicos (Rovinski, 2007; Silva, 2005). O segundo, por sua vez, relaciona-se a uma lesão ao funcionamento psicológico, seja esta parcial ou global, que afeta as esferas afetivas ou intelectuais, podendo ou não resultar em um diagnóstico psiquiátrico, mas que, necessariamente, implica num prejuízo das aptidões psíquicas prévias, cabendo aos técnicos da saúde mental a sua avaliação (Castex, 1997 citado por Rovinski, 2007). Todavia, ambos se assemelham por não poderem prescindir do discurso jurídico e por estarem intimamente relacionados ao direito de reparação/ressarcimento de alguma perda sofrida (Castex, 1997 citado por Rovinski, 2007).

Uma das maiores dificuldades encontradas na avaliação de uma psicopatologia decorrente de acidente de trabalho é confirmar se aquele dano constatado possui, de fato, relação direta com o evento traumático experienciado, ou seja, se aquela situação vivenciada no ambiente de trabalho foi o fator desencadeante da psicopatologia (Dorigo \& Lima, 2007; Jacques, 2007; Lima, 2005; Rovinski, 2007; Vieira, 2009). Portanto, um dos empecilhos em termos de constatação diagnóstica refere-se à falta de prejuízo materialmente visível nos casos dos traumas psíquicos, o que contribui para a dificuldade de se mensurar a dimensão da lesão causada.

O TEPT é um dos únicos transtornos mentais que permite estabelecer uma relação de causalidade direta com o trabalho (Dorigo \& Lima, 2007; Hensel et al., 2010; McFarlane \& Bryant, 2007; Ministério da Saúde do Brasil, 2001), tendo em vista a necessidade da experiência, direta ou indireta, de um evento estressor traumático para a sua constatação (APA, 2002). Não obstante, o seu diagnóstico não é tarefa simples, exigindo minuciosidade na investigação de seus sintomas, incluindo análise da história pregressa do indivíduo, diagnóstico diferencial entre outros possíveis quadros psicopatológicos e, o mais importante, o nexo causal entre o evento traumático experienciado e os sintomas pós-traumáticos examinados (Dorigo \& Lima, 2007). A partir da avaliação clínica realizada e da causalidade estabelecida, deverá ser possível mensurar as consequências da situação traumática vivenciada, incluindo questões como incapacidade temporária ou permanente para o trabalho e projeções futuras em termos de necessidade de tratamentos e compensação (Miller, 2001).

\section{Considerações finais}

Nas situações de doenças relacionadas ao trabalho, uma análise isolada da estrutura e de traços de personalidade é, muitas vezes, insuficiente no entendimento dos reais motivos que desencadearam determinados transtornos mentais (Vieira, 2009). Desse modo, é imprescindível a realização de uma avaliação complexa e imparcial da interação indivíduo/ambiente 
de trabalho na determinação de distúrbios mentais decorrentes de acidentes de trabalho.

Dessa maneira, considerando as implicações clínicas, socioeconômicas e legais decorrentes da vivência de um evento traumático, incluindo os aspectos que podem motivar uma ação indenizatória por acidente de trabalho, um julgamento assertivo sobre as reais condições do trabalhador demonstrase imprescindível. Uma avaliação clínica criteriosa e acurada revela-se extremamente necessária tanto para a determinação de um diagnóstico assertivo e específico quanto para auxiliar, posteriormente, na elaboração de um tratamento adequado, considerando as particularidades de cada transtorno e desenvolvendo intervenções terapêuticas eficazes.

Conforme Teixeira (2007), a Emenda Constitucional 45/2004, referente ao julgamento das ações indenizatórias por acidente de trabalho e doenças ocupacionais, incita para a reflexão acerca da proteção jurídica da saúde do trabalhador. Uma das questões que impera diante da categorização de um transtorno mental como decorrente de acidente de trabalho, conforme mencionado anteriormente, diz respeito ao estabelecimento da relação de causalidade, ou seja, se aquela situação vivenciada durante a jornada de trabalho foi o desencadeante de determinado dano psíquico. Especificamente em relação ao TEPT, a classificação adotada no Brasil para a categorização das doenças relacionadas ao trabalho asseguraria uma relação causal direta e imediata entre o transtorno e o trabalho, que constitui causa necessária (Ministério da Saúde do Brasil, 2001).

O papel da empresa e das entidades sindicais como fontes de apoio social após a vivência de uma experiência traumática no ambiente de trabalho parece ser tão importante quanto a prestação de outros serviços profissionais especializados. O investimento na saúde do trabalhador, por parte das empresas, beneficia os sistemas de saúde pública e previdenciário, os rendimentos da organização e até mesmo a imagem desta, pois os efeitos da violência nesse ambiente diminuem o nível da produtividade e aumentam o absenteísmo (Mossink, 2004; Steffgen, 2008). Portanto, melhorar as condições de trabalho, investir em segurança e em treinamento adequado, fornecer acesso ao sistema de saúde e suporte aos funcionários são algumas das medidas que podem amenizar a ocorrência de experiências traumáticas nos contextos de trabalho, servindo como fatores de prevenção primária, pois impedem o aparecimento da doença (Hensel et al., 2010; McFarlane \& Bryant, 2007).

Entre os empregados que sofreram algum trauma, convém ressaltar para a importância do acolhimento e do encaminhamento a tratamento especializado logo após o acontecimento do evento traumático, com políticas de planejamento para lidar com essas consequências, a fim de evitar o desencadeamento e a cronificação do TEPT (Hensel et al., 2010; Miller, 2000). Essas medidas são consideradas efetivas, já que é bem estabelecido na literatura que a falta de apoio social é um fator preditivo para o TEPT (Brewin, Andrews, \& Valentine, 2000; Keane et al., 2006; Laffaye, Cavella, Drescher, \& Rosen, 2008; Steffgen, 2008). A prevenção do TEPT no ambiente de trabalho requer uma abordagem interdisciplinar, o que inclui uma ação articulada entre os sistemas assistenciais e de vigilância, de modo que estejam capacitados e preparados para lidar com esse tipo de acidente, atentando tanto ao sofrimento psíquico, como aos aspectos sociais e de intervenção nos contextos organizacionais (Ministério da Saúde do Brasil, 2001; Seligmann-Silva, 1997).

Autores destacam que alguns sinais apresentados pelos funcionários podem ser facilmente observados pelos empregadores e dizem respeito à presença de fatores de risco que podem predizer o desenvolvimento do TEPT frente a um evento traumático, como desorganização, agitação psicomotora, tensão, uso de substâncias, agressividade, entre outros (Hensel et al., 2010; Miller, 2000; U.S. Department of Justice, 2002). Esses fatores atentam para a importância de uma ação preventiva de cunho secundário ao TEPT, pois envolve a detecção de sinais precoces do transtorno (Hensel et al., 2010; Miller, 2001).

A partir da revisão da literatura apresentada, fica evidente a repercussão do TEPT para a vida do indivíduo, com prejuízos significativos nas relações familiares, sociais e, sobretudo, no ambiente de trabalho. Desse modo, a questão sobre o TEPT decorrente de acidente de trabalho, especificamente, suscita para a consideração em torno de diferentes eixos: a prevenção, a avaliação, o tratamento e o ressarcimento acerca dos prejuízos causados. Entretanto, para o cumprimento de tais ações, revelase imprescindível um conhecimento aprofundado sobre o que é o TEPT, quais são as suas causas e as suas consequências para as vítimas acometidas pelo transtorno. Para isso, pesquisas que deem conta desses aspectos são extremamente necessárias, a fim de que a compreensão sobre o transtorno se torne cada vez mais clara, favorecendo a implementação de medidas preventivas e terapêuticas mais eficazes.

\section{Referências}

Associação Americana de Psiquiatria (2002). Manual diagnóstico e estatístico de transtornos mentais (4a . Ed.). Porto Alegre: Artmed.

Beck, J. G., Grant, D. M., Clapp, J. D., \& Palyo, S. A. (2009). Understanding the interpersonal impact of trauma: contributions of PTSD and depression. Journal of Anxiety Disorders, 23, 443-450.

Berger, W., Figueira, I., Maurat A., Bucassio, E., Vieira, I., Jardim, S., ... Mendlowicz, M. V. (2007). Partial and full PTSD in Brazilian ambulance workers: prevalence and impact on health and on quality of life. Journal of Traumatic Stress, 20, 637-642.

Berninger, A., Webber, M. P., Cohen, H. W., Gustave, J., Lee, R., Niles, J. K., ... Prezant, D. J. (2010). Trends of elevated PTSD risk in firefighters exposed to the World Trade Center disaster: 2001-2005. Public Health Reports, $125,556-566$

Breslau, N., Chilcoat, H., Kessler, R., \& Davis, G. (1999). Previous exposure to trauma and PTSD effects of subsequent trauma: results from the Detroit area survey of trauma. American Journal Psychiatry, 156, 902-907.

Brewin, C. R. (2007). Remembering and forgetting. In M. J. Friedman, T. M. Keane \& P. A. Resick (Orgs.), Handbook of PTSD: Science and practice (pp. 116-124). Nova Iorque: Guildford Press.

Brewin, C. R., Andrews, B., \& Valentine, J. D. (2000). Meta-analysis of risk factors for posttraumatic stress disorder in trauma-exposed adults. Journal of Consulting and Clinical Psychology, 68, 748-766.

Bucasio, E., Vieira, I., Berger, W., Martins, D., Souza, C., Maia, D., ... Jardim, S. (2005). Transtorno de estresse pós-traumático como acidente de trabalho em um bancário: relato de um caso. Revista de Psiquiatria do Rio Grande do $\mathrm{Sul}, 27,86-89$.

Buodo, G., Ghisi, M., Novara, C., Scozzari, S., Di Natale, A., Sanavio, E., \& Palomba, D. (2011). Assessment of cognitive functions in individuals with 
post-traumatic symptoms after work-related accidents. Journal of Anxiety Disorders, 25, 64-70.

Cacciola, J. S., Koppenhaver, J. M., Alterman, A. I., \& McKay, J. R. (2009). Posttraumatic stress disorder and other psychopathology in substance abusing patients. Drug and Alcohol Dependence, 101, 27-33.

Chamon, E. M. Q. O. (2006). Estresse e estratégias de enfrentamento: o uso da escala Toulousaine no Brasil. Revista Psicologia: Organizações e Trabalho, 6(2), 43-64.

Cohidon, C., Diène, E., Carton, M., Fatras, J., Goldberg, M., \& Imbernon, E. (2009). Mental health of workers in Toulouse 2 years after the industrial AZF disaster: first results of a longitudinal follow-up of 3,000 people. Social Psychiatry and Psychiatric Epidemiology, 44, 784-791.

Constituição da República Federativa do Brasil de 1988 (1988, 5 de outubro). Diário Oficial da União.

Dantas, H. S. \& Andrade, A. G. (2008). Comorbidade entre transtorno de estresse pós-traumático e abuso e dependência de álcool e drogas: uma revisão da literatura. Revista de Psiquiatria Clínica, 35(1), 55-60.

De Mol, J. (1998). Post-aggression stress disorders: psychosocial and work repercussions. Medical Science Monitor, 5, 277-282.

Dorigo, J. N., \& Lima, M. E. A. (2007). O transtorno de estresse pós-traumático nos contextos de trabalho: reflexões em torno de um caso clínico. Cadernos de Psicologia Social do Trabalho, 10, 55-73.

Duarte, E. A. (2002). Teoria geral do crime no ordenamento jurídico brasileiro. Campo Grande: UCDB.

Einav, S., Shalev, A., Ofek, H., Freedman, S., Matot, I., \&. Weiniger, C. (2008). Differences in psychological effects in hospital doctors with and without posttraumatic stress disorder. The British Journal of Psychiatry, 193, 165-166.

Foa, E. B., Keane, T. M., \& Friedman, M. J. (2000). Introduction. In E. B. Foa, T. M. Keane \& M. J. Friedman (Orgs.), Effective treatments for PTSD: practice guidelines from the international society for traumatic stress studies (pp. 1-17). Nova Iorque: Guilford Press.

Green, J. G., McLaughlin, K. A., Berglund, P. A., Gruber, M. J., Sampsom, N. A., Zaslavsky, A. M., \& Kessler, R. C. (2010). Childhood adversities and adult psychopathology in the National Comorbidity Survey Replication (NCS-R) I: associations with first onset of DSM-IV disorders. Archives of General Psychiatry, 67, 113-123.

Hensel, J., Bender, A., Bacchiochi, J., Pelletier, M., \& Dewa, C. S. (2010). A descriptive study of a specialized worker's psychological trauma program. Occupational Medicine, 60, 654-657.

Hernández, P., Gangsei, D., \& Engstrom, D. (2007). Vicarious resilience: a new concept in work with those who survive trauma. Family Process, 46, 229-241.

Jacques, M. G. (2007). O nexo causal em saúde/doença mental no trabalho: uma demanda para a Psicologia. Psicologia \& Sociedade, 19, 112-119.

Keane, T. M., Marshall, A. D., \& Taft, C. T. (2006). Posttraumatic stress disorder: etiology, epidemiology, and treatment outcome. Annual Review of Clinical Psychology, 2, 161-197.

Kessler, R. C. (2000). Posttraumatic stress disorder: the burden to the individual and to the society. Journal of Clinical Psychiatry, 61, 4-12.

Kessler, R. C., Berglund, P., Demler, O., Jin, R., Merikangas, K., \& Walters, E. (2005). Lifetime prevalence and age-of-onset distributions of DSM-IV disorders in the National Comorbidity Survey Replication. Archives of General Psychiatry, 62, 593-602.

Kessler, R. C., Chiu, W. T., Demler, O., Merikangas, K. R., \& Walters, E. E. (2005). Prevalence, severity, and comorbidity of 12-month DSM-IV disorders in the National Comorbidity Survey Replication. Archives of General Psychiatry, 62, 617-627.

Kessler, R. C., Hwang, I., LaBrie, R., Petukhova, M., Sampson, N. A., Winters, K. C., \& Shaffer, H. J. (2008). DSM-IV pathological gambling in the National Comorbidity Survey Replication. Psychological Medicine, 38, 1351-1360.

Kessler, R. C., Sonnega, A., Bromet, E., Hughes, M., \& Nelson, C. B. (1995). Posttraumatic stress disorder in the National Comorbidity Survey. Archives of General Psychiatry, 52, 1048-1060.
Knapp, P., \& Caminha, R. M. (2003). Terapia cognitiva do transtorno de estresse pós-traumático. Revista Brasileira de Psiquiatria, 25, 31-36.

Kristensen, C. H., Parente, M. A. M. P., \& Kaszniak, A. W. (2005). Transtorno de estresse pós-traumático: critérios diagnósticos, prevalência e avaliação. In R. M. Caminha (Org.), Transtornos do estresse pós-traumático (TEPT): da neurobiologia à terapia cognitiva (pp. 15-35). São Paulo: Casa do Psicólogo.

Laffaye, C., Cavella, S., Drescher, K., \& Rosen C. (2008). Relationships among PTSD symptoms, social support, and support source in veterans with chronic PTSD. Journal of Traumatic Stress, 21, 394-401.

Lei no 8.213, de 24 de julho de 1991 (1991, 24 de julho). Dispõe sobre os Planos de benefícios da previdência social e dá outras providências. Diário Oficial da União.

Lei ${ }^{\circ} 10.406$, de 10 de janeiro de 2002 (2002, 10 de janeiro). Institui o Código Civil. Diário Oficial da União.

Lei $\mathrm{n}^{\circ}$ 11.340, de 26 de dezembro de 2006 (2006, 26 de dezembro). Diário Oficial da União.

Lima, M. E. A. (2005). Transtornos mentais e trabalho: O problema do nexo causal. Revista de Administração da FEAD-Minas, 2, 73-80.

Lydiard, R. B., \& Hamner, M. H. (2009). Clinical importance of sleep disturbance as a treatment target in PTSD. Spring, 7, 176-183.

MacDonald, H. A., Colotla, V., Flamer, S., \& Karlinsky, H. (2003). Posttraumatic stress disorder (PTSD) in the workplace: a descriptive study of workers experiencing PTSD resulting from work injury. Journal of Occupational Rehabilitation, 13, 63-77.

Maia, D. B., Marmar, C. R., Metzler, T., Nóbrega, A., Berger, W., Mendlowicz, M. V.,...Figueira, I. (2007). Post-traumatic stress symptoms in an elite unit of Brazilian police officers: prevalence and impact on psychosocial functioning and on physical and mental health. Journal of Affective Disorders, 97, 241-245.

Margis, R. (2003). Comorbidade no transtorno de estresse pós-traumático: regra ou exceção? Revista Brasileira de Psiquiatria, 25, 17-20.

Matthews, L. R., Harris, L. M., \& Cumming, S. (2009). Trauma-related appraisals and coping styles of injured adults with and without symptoms of PTSD and their relationship to work potential. Disability and Rehabilitation, 31, 1577-1583.

McFarlane, A. C., \& Bryant, R. A. (2007). Post-traumatic stress disorder in occupational settings: anticipating and managing the risk. Occupational Medicine, 57, 404-410.

Mendes, R., \& Dias, E. C. (1991). Da medicina do trabalho à saúde do trabalhador Revista de Saúde Pública, 25, 341-349.

Miller, L. (2000). Law enforcement traumatic stress: clinical syndromes and intervention strategies. Trauma Response, 6, 15-20.

Miller, L. (2001). Workplace violence and psychological trauma: clinical disability, legal liability, and corporate policy. Part I. Neurolaw Letter, 11, 1-5.

Ministério da Previdência Social (2010). Recuperado de http://www. previdenciasocial.gov.br/conteudoDinamico.php?id=39

Ministério da Previdência Social, Empresa de Tecnologia e Informações da Previdência Social (2009). Anuário Estatístico da Previdência Social. Brasília, 17: MPS/DATAPREV.

Ministério do Trabalho e Emprego, \& Ministério da Previdência Social (2008) Anuário Estatístico de Acidentes do Trabalho: AEAT 2007, 1. Brasília: MTE: MPS.

Ministério do Trabalho e Emprego, \& Ministério da Previdência Social (2009). Anuário Estatístico de Acidentes do Trabalho: AEAT 2008, 1. Brasília: MTE: MPS.

Ministério da Saúde do Brasil (2001). Doenças relacionadas ao trabalho: Manual de procedimentos para os serviços de saúde. Ministério da Saúde do Brasil, Organização Pan-Americana da Saúde no Brasil. Brasília: Ministério da Saúde do Brasil.

Monson, C. M., \& Friedman, M. J. (2006). Back to the future of understanding trauma: implications for cognitive-behavioral therapies for trauma. In V. M. Follette \& J. I. Ruzek. (Orgs.), Cognitive-behavioral therapies for trauma 
(pp. 1-17). Nova Iorque: Guilford Press.

Mossink, J. (2004). Comprender y aplicar el analisis económico en la empresa. Série protección de la salud de los trabajadores. Francia: Organización Mundial de la Salud.

Murofuse, N. T., \& Marziale, M. H. P. (2001). Mudanças no trabalho e na vida de bancários portadores de lesões por esforços repetitivos: LER. Revista Latino-Americana da Enfermagem, 9(4), 19-25.

Nock, M. K., Hwang, I., Sampson, N., Kessler, R. C., Angermeyer, M., Beautrais, A., ... Williams, D. R. (2009). Cross-national analysis of the associations among mental disorders and suicidal behavior: findings from the World Mental Health Surveys. PLoS Medicine, 6, 1-17.

Novara, C., Buodo, G., Ghisi, M., Poli, S., Bichescu, D. M., Sanavio, E., \& Palomba, D. (2009). Integrated assessment of emotional distress after work - related accidents. Stress and Health, 25, 195-204.

Olatunji, B. O., Cisler, J. M., \& Tolin, D. F. (2007). Quality of life in the anxiety disorders: a meta-analytic review. Clinical Psychology Review, 27, 572-581.

Organização Mundial da Saúde. (1993). Classificação dos transtornos mentais e de comportamento da CID-10: descrições clínicas e diretrizes diagnósticas. Porto Alegre: Artes Médicas.

Ouimette, P., Read, J. P., Wade, M., \& Tirone, V. (2010). Modeling associations between posttraumatic stress symptoms and substance use. Addictive Behaviors, 35, 64-67.

Palácios, M., Duarte, F., \& Câmara, V. M. (2002). Trabalho e sofrimento psíquico de caixas de agências bancárias na cidade do Rio de Janeiro. Cadernos de Saúde Pública, 18, 843-851.

Remédio, J. A., Freitas, J. F. S., \& Junior, J. J. L. (2000). Dano moral: Doutrina, jurisprudência e legislação. São Paulo: Saraiva.

Richards, D. (2000). Brief report Symptom severity, personal and social variables after armed robbery. British Journal of Clinical Psychology, 39, 415-419.

Rovinski, S. L. R. (2007). Fundamentos da Perícia Psicológica Forense. São Paulo: Vetor.

Rubin, D. C., Berntsen, D., \& Johansen, M. K. (2008). A memory based model of posttraumatic stress disorder: evaluating basic assumptions underlying the PTSD diagnosis. Psychological Review, 115, 985-1011.

Schnurr, P., Green B., \& Kaltman, S. (2007). Trauma exposure and pshysical health. In M. J. Friedman, T. M. Keane \& P. A. Resick (Orgs.), Handbook of PTSD: Science and practice (pp. 406-424). Nova Iorque: Guilford Press. Seligmann-Silva, E. (1997). Saúde mental e automação: a propósito de um estudo de caso no setor ferroviário. Cadernos de Saúde Pública, 13, 95-109.

Sher, L. (2004). Recognizing post-traumatic stress disorder. QJ Med, 97(1), 1-5. Shercliffe, R., \& Colotla, V. (2009). MMPI-2 profiles in civilian PTSD: an examination of differential responses between victims of crime and industrial accidents. Journal of Interpersonal Violence, 24, 349-360.

Silva, A. L. M. (2005). O dano moral e a sua reparação civil. São Paulo: Editora Revista dos Tribunais.

Steffgen, G. (2008). Physical violence at the workplace: consequences on health and measures of prevention. Revue Européenne de Psychologie Appliquée, $58,285-295$.

Taylor, S. (2006). Clinican's guide to PTSD: a cognitive-behavioral approach. Nova Iorque: Guilford Press.

Teixeira, S. (2007). A depressão no meio ambiente do trabalho e sua caracterização como doença do trabalho. Revista Tribunal Regional do Trabalho $3^{a}$ Região, 46(76), 27-44.

U.S. Department of Justice (2002). Workplace Violence Issues in Response. Federal Bureau of Investigation, Critical Incident Response Group, National Center for the Analysis of Violent Crime. FBI Academy, Quantico, Virginia.

Van Loey N., Son, M., Heijden, P., \& Ellis, I. (2008). PTSD in persons with burns: an explorative study examining relationships with attributed responsibility, negative and positive emotional states. Burns, 34, 1082-1089.

Vieira, C. E. C. (2009). O nexo causal entre transtorno de estresse pós-traumático e trabalho: controvérsias acerca do laudo de uma perícia judicial. Revista Brasileira de Saúde Ocupacional, 34(120), 150-162.

Walker, E., Katon, W., Russo, J., Ciechanowski, P., Newman, E., \& Wagner, A. (2003). Health care costs associated with posttraumatic stress disorder symptoms in women. Archives of General Psychiatry, 60, 369-374.

Wells, A., Welford, M., Fraser, J., King, P., Mendel, E., Wisely, J., ... Rees, D. (2008). Chronic PTSD treated with metacognitive therapy: an open trial. Cognitive and Behavioral Practice, 15, 85-92.

Wolf, S. (2009). The year in burns 2008. Burns, 35, 1057-1070.

World Health Organization. (1998). Enciclopedia de salud y seguridad en el trabajo. Edición española. Ministerio de Trabajo y Asuntos Sociales.

Luiziana Souto Schaefer, mestre em Psicologia pela Pontifícia Universidade Católica do Rio Grande do Sul, doutoranda em Psicologia pela Pontifícia Universidade Católica do Rio Grande do Sul, é perita criminal pelo Instituto Geral de Perícias do Rio Grande do Sul. Endereço para correspondência: Avenida Ipiranga, 6681, Partenon, Porto Alegre-RS. CEP: 90619-900. Telefone:(51)33203500 - Ramal: 7741e Fax: (51)33203633. E-mail: luiziana.schaefer@gmail.com

Beatriz de Oliveira Meneguelo Lobo, é psicóloga e mestranda pela Pontifícia Universidade Católica do Rio Grande do Sul. E-mail: beatrizlobo.m@gmail.com

Christian Haag Kristensen, doutor em Psicologia pela Universidade Federal do Rio Grande do Sul, é professor do Programa de Pós-Graduação em Psicologia da Pontifícia Universidade Católica do Rio Grande do Sul, é professor do curso de Psicologia na Faculdade de Psicologia do Rio Grande do Sul. E-mail: christian. kristensen@pucrs.br 